\title{
Masking of tones by tones and of noise by noise
}

\author{
DONALD E. ROBINSON \\ Indiana University, Bloomington, Indiana 47401 \\ TED L. LANGFORD \\ Purdue University, West Lafayette, Indiana 47907 \\ and \\ WILLIAM A. YOST \\ University of Florida, Gainesville, Florida 32605
}

\begin{abstract}
Using a two-alternative temporal forced-choice technique, two binaural detection experiments were performed. In the first, the detectability of a $250-\mathrm{Hz} 128$-msec tonal signal masked by a gated 70-dB SPL tone of the same frequency and duration was measured as a function of the level of the signal, the phase angle at which the signal was added to the masker, and the interaural phase difference of the signal. In the second experiment, the signal was a wideband $(100-3,000 \mathrm{~Hz}) 128-\mathrm{msec}$ Gaussian noise masked by a continuous Gaussian noise of the same bandwidth and coherent with the signal. The detectability of this noise signal was measured as a function of the same variables investigated in the first experiment. In both experiments detectability was found to follow a simple energy-or power-detection model when the interaural phase difference was $0 \mathrm{deg}$. When the interaural phase difference was $180 \mathrm{deg}$, the function relating the signal level required for a constant level of performance to the signal-masker phase angle is such that neither the Webster-Jeffress hypothesis nor Durlach's E-C model accounts for the data. The data are reasonably well fit by a model proposed by Hafter and Carrier.
\end{abstract}

In 1956, Jeffress, Blodgett, Sandel, and Wood published a comprehensive paper on masking. That report included a review of the literature of binaural and monaural masking, models for monaural and binaural detection, and new results for both monaural and binaural detection. Of particular importance to the present paper are the model for binaural signal detection and the data on tone-on-tone masking under antiphasic conditions.

The model of binaural signal detection suggested by Jeffress, Blodgett, Sandel, and Wood is a combination of Webster's (1951) phase- or time-shift model and Jeffress's (1948) neural delay-line model. Briefly, Webster suggested that, for tonal signals, noise maskers, and dichotic listening conditions, the interaural time shift which occurs at the signal frequency when the tone is added to the noise is the primary cue for binaural signal detection. Jeffress's neural delay line provides a device which can sense the time shifts generated under dichotic conditions and, thus, is the processor which determines the advantages of binaural listening over monaural.

Prior to the Jeffress et al paper, an embarrassing observation for the Webster-Jeffress hypothesis was that binaural advantages were not found with tonal maskers and tonal signals. Hirsh and Webster (1949) cite a personal communication from Licklider in which Licklider was unable to obtain a release from masking with an antiphasic condition when both signal and masker were tones. Hirsh and Webster investigated many types of maskers (tones, pulses, and noises of various bandwidths) and failed to find an improvement in antiphasic conditions over homophasic ones when both signal and masker were tones of the same frequency.
However, Hirsh and Webster apparently did not manipulate the phase angle at which the signal was added to the masker. Jeffress et al, by using tones of short duration and by careful selection of the signal-masker phase angle, were able to obtain a sizable improvement with a tonal signal under antiphasic conditions.

Since 1956, and undoubtedly due to the Jeffress, Blodgett, Sandel, and Wood paper, a large amount of work has been done on binaural masking phenomena (see Green \& Henning, 1969). Most of this work has involved the masking of tonal signals by noise maskers. Under such conditions, the Webster-Jeffress time-shift model can be tested only through statistical arguments, since the phase angle between signal and masker is not static, but is constantly changing. Such statistical arguments (Robinson, 1966; Levitt \& Lundry, 1966) have lent some support to the model, but cannot be considered critical due to the many assumptions which are necessary. Thus, conditions which allow control over the signal-masker phase are necessary before the time-shift model can be properly tested. Two such conditions are the masking of tones by tones and of noise by coherent noise.

A second important model for binaural signal detection is the Durlach equalization-cancellation (E-C) model (Durlach, 1972). This model proposes that the binaural processing system consists of (1) a transform of the masker inputs to the ears such that they are made as nearly identical as possible (equalization) and (2) subtraction of the equalized waveform at one ear from that at the other (cancellation). After these operations, a decision is made on the basis of the power of this difference waveform. The model includes two 
free parameters in the form of internal noise (time jitter and amplitude jitter). The Durlach model is extremely accurate and general in its prediction of binaural masking phenomena when the inputs are tonal signals and noise maskers.

Several papers since 1956 have reported attempts to control the signal-masker phase relations. The first (Rilling \& Jeffress, 1965) made use of a single noise source for both signal and masker. The signal was narrowband filtered and phase shifted relative to the wideband masker. Interaural phase relations of $0 \mathrm{deg}$ (SO) and $180 \mathrm{deg}\left(\mathrm{S}_{\mathrm{n}}\right)$ were investigated with a homophasic noise masker (MO) at several values of signal-masker phase $(\alpha)$. Large advantages of dichotic listening over diotic (11.5 to $18.0 \mathrm{~dB})$ were found as $\alpha$ was varied from 0 to $180 \mathrm{deg}$. However, the improvement was a function of deteriorating performance under MO-SO (the diotic conditions), and not due to improved performance under $\mathrm{MO}-\mathrm{S}_{\Pi}$ (dichotic). In fact, the signal level necessary for a constant level of performance under $S_{\Pi}$ was invariant as $\alpha$ was varied. Rilling and Jeffress concluded that this result was due to a technical problem: the effect of the phase shifts introduced by the filter in the signal channel on the interaural phase relations under $\mathrm{MO}_{\Pi}$. The explanation, in their words, is that "the interaural phase shifts for the composite narrow band of noise will be almost independent of the settings of the phase shifter." Because of this problem, the experiment has not been considered a valid test of either the Webster-Jeffress hypothesis or of the Durlach model. It should be noted, however, that the results for $S_{\Pi}$ were exactly those predicted by the E-C model: $S_{\Pi}$ detection was independent of the angle $\alpha$.

The second experiment to control the signal-masker phase angle is one by Wightman (1969). Wightman used a tonal masker of $250 \mathrm{~Hz}$ and a tonal signal of the same frequency and extended the work of Jeffress, Blodgett, Sandel, and Wood. When the masker was presented continuously, as in the Jeffress et al paper, Wightman found an advantage of $S_{\Pi}$ over $S O$ of about $12 \mathrm{~dB}$ when $\alpha$ was $90 \mathrm{deg}$, but an advantage of SO over $S_{\Pi}$ of about $3 \mathrm{~dB}$ when $\alpha$ was $0 \mathrm{deg}$. However, in this portion of the research, the SO condition was confounded by energy spread caused by the phase shift induced when the signal was added to the masker. In a second experiment, Wightman used a gated masker in order to avoid the effects of the phase-induced energy spread. Under these conditions, the SO function was that expected uncier a constant power- or energy-increment hypotnesis. However, with the gated masker the $S_{11}$ condition always required a greater signal level than did the SO condition, i.e., diotic detection was always better than dichotic. The data from both the continuous and pulsed experiments showed that, for $S_{\Pi}$, the signal level necessary for $75 \%$ correct detections is independent of the value of $\alpha$.

Wightman points out that the results of both of his experiments contradict predictions made by the Webster-Jeffress model. The model predicts that as $\alpha$ is varied, detectability under the $S_{\text {II }}$ condition should change, with maximum detectability occurring when $\alpha$ is $90 \mathrm{deg}$, since, at this value, the interaural phase difference is at a maximum. The Durlach model, since it posits the power of the difference in the equalized waveforms as the decision variable, predicts that $S_{\Pi}$ detectability will be independent of the signal-masker phase angle, the result obtained by Wightman. However, the formal statement of the Durlach model precludes dichotic detection being worse than diotic, since it assumes that when binaural detection is worse than monaural, the system will use the best monaural channel. Wightman found diotic detection better than dichotic. Thus, the data cannot be taken as support for Durlach's model.

A third experiment involving fixed signal-to-masker phase angles is that of Hafter and Carrier (1970). Their experiment, like that of Wightman, involved tonal maskers and tonal signals. Both masker and signal were pulsed during the observation intervals of a "same-different" experimental paradigm. They investigated three frequencies $(250,500$, and $1,000 \mathrm{~Hz})$ and four values of signal-to-masker phase angle $(0,45$, 90 , and $135 \mathrm{deg})$. Their data show considerable inter-S variability, but two general conclusions can be drawn: detectability for $S_{\Pi}$ signals is poor at $\alpha=0$ deg and at $\alpha$ $=45 \mathrm{deg}$. and the $\alpha=135 \mathrm{deg}$ condition in which the interaural temporal cue is in opposition to the interaural intensive cue tends to produce poorest performance. Hafter and Carrier suggest that over the range of 0 to $90 \mathrm{deg}$, their data are reasonably well fit by a "lateralization" model which assumes that the observer is making his decision on the basis of a weighted sum of the interaural temporal and interaural intensive cues. (The weighting may be thought of as converting interaural intensive difference into temporal difference by multiplying the intensive difference by a "trading relation" expressed in microseconds/decibel.) Such a model fits less well at $135 \mathrm{deg}$ where the interaural cues are in opposition.

Yost $(1972 \mathrm{a}, \mathrm{b})$ has also studied binaural tonal masking. In one study (1972a) he investigated the SO and $S_{\Pi}$ conditions at three $\alpha s(0,45$, and $90 \mathrm{deg})$ as a function of a 12-dB change in the intensity of the masker. Yost's data were obtained at $500 \mathrm{~Hz}$ and were similar to those obtained by Hafter and Carrier (1969) at $500 \mathrm{~Hz}$. Yost reported that the signal-to-masker ratio required for detection remained constant in both the SO and $S_{n}$ conditions over the $12-\mathrm{dB}$ change in masker level. In the other study, Yost (1972b) investigated the $\mathrm{SO}, \mathrm{S}_{\Pi}$, and the SM conditions. In the SM condition the tonal signal was added only to one ear while the tonal masker was diotic. His $S O$ and $S_{\Pi}$ data at 250 and $500 \mathrm{~Hz}$ are similar to Hafter and Carrier's data at 250 and $500 \mathrm{~Hz}$. At $3,000 \mathrm{~Hz}$, Yost reported no difference in detection between the $\mathrm{SO}$ and $\mathrm{S}_{\Pi}$ conditions. At all 
three $\alpha$ s $(0,45$, and $90 \mathrm{deg})$ and for 250 and $500 \mathrm{~Hz}$, the SM condition yielded larger values of the signal-to-masker ratio required for detection than the $S_{\Pi}$ condition. At $3,000 \mathrm{~Hz}$ there was no difference reported among the SO, $S_{\Pi}$, and $S M$ conditions at $\alpha$ of 45 and $90 \mathrm{deg}$. However, at an $\alpha$ of $0 \mathrm{deg}$ the SM condition yielded poorer detection (average of $5 \mathrm{~dB}$ ) than the SO condition at all three frequencies $(250,500$, and $3,000 \mathrm{~Hz}$ ). Yost concluded in both studies that the lateralization model provides a better fit to the data than either the Webster-Jeffress or Durlach models.

Jeffress and McFadden (1971) and McFadden, Jeffress, and Ermey (1971) have reported data from experiments in which both signal and masker are obtained by passing a Gaussian noise through a narrow $(50-\mathrm{Hz})$ filter. In the Jeff ress and McFadden experiment, the filter was centered at $500 \mathrm{~Hz}$ and in that by McFadden et al at $250 \mathrm{~Hz}$. Both of these studies investigated the lateralizability as well as the detectability of the noise signals as a function of the signal-to-masker phase angle. As was the case in the Hafter and Carrier experiment, both of these reports show considerable inter-S variability. With respect to the detection data, however, two general conclusions may be drawn: (1) neither a strict time-cue model (i.e., Webster-Jeffress) nor the E-C model seems to fit the data, and (2) when the temporal and intensive cues are in opposition, performance tends to be worse than when they are in agreement.

Wightman (1971) has reported an extensive investigation of the effects of masker bandwidth on the detectability of SO and $\mathrm{S}_{\Pi}$ signals. The main conclusion of the paper is that the spectral content of both signal and masker may have large effects on the detection of SO signals, while such effects are relatively small when the signals are $S_{\Pi}$. In this paper, Wightman also presents data from an experiment similar to the one reported by him in 1969 with continuous sine wave maskers. In the more recent experiment, however, the sine wave was heavily filtered to avoid the problerrs caused by transients in the earlier work. Wightman applies the "lateralization" model described by Hafter and Carrier to the tone-on-tone data at 262 and $800 \mathrm{~Hz}$. Although the model somewhat overestimates performance for the $S_{\Pi}$ data, it provides a reasonably good fit at both frequencies.

Since 1956, then, there have been nine experiments which have controlled the signal-to-masker phase angle and have investigated detectability for $S_{\Pi}$ signals: six of these papers have employed tonal maskers and tonal signals and three have employed a noise as both masker and signal. The most striking observation one can make about these papers is that inter-S and interexperiment differences are so large that a consistent picture of the effects of varying signal-to-masker phase angle on dichotic signal detection has failed to emerge.

Three basic models have been described and applied to the controlled phase experiments. The first of these, the Webster-Jeffress hypothesis, proposes interaural temporal difference as the primary cue for binaural signal detection. This model, which has had considerable success in handling the data of tone-in-noise experiments, fails in almost all of the experiments described here, since it predicts best detection at a signal-masker phase angle of $90 \mathrm{deg}$, a result which rarely occurs. The second model, the Durlach E-C model, has also been applied to tone-in-noise masking and, for most such conditions, provides a reasonably good fit to the data. When applied to experiments in which the signal-masker phase angle is controlled, the model predicts that detection under antiphasic conditions will be independent of signal-to-masker phase. Although some $\mathrm{Ss}$ in some experiments provide data in which the effect of varying signal-to-masker phase is small, the consensus appears to be that the model fails. Certainly, some data show large effects of varying signal-to-masker phase. A third model, the "lateralization" model proposed by Hafter and Carrier (a similar model was also proposed by Yost, 1970), has only recently been applied to tone-in-noise masking (Hafter, 1972), and it is perhaps too early to say how it will fare. This model, however, appears to provide the best fit of any of the models to tone-on-tone masking. However, as Hafter and Carrier point out, the model provides a poor fit when the interaural temporal and interaural intensive cues are in opposition.

Binaural masking studies using coherent signals and maskers allow for the control of the signal-to-masker phase angle. This control is not possible in studies in which a pulsed tonal signal and continuous broadband noise are used. The control of the signal-to-masker phase angle in the coherent signal and masker studies allows one to make predictions regarding the significance of the phase angle in studies involving tonal signals and noise maskers. However, in order to make these predictions, the waveforms in these two types of studies should be as similar as possible. Only sinusoids and narrowband noise have been used to study binaural masking with coherent signals and maskers. Sinusoidal signals and maskers allow for the control of the signal-to-masker phase angle, but the masker must be pulsed with the signal to avoid spread of energy (Wightman, 1972). Narrowband noise signals and maskers also allow for control of $\alpha$, and the noise may be continuous while the signal is pulsed. However, the narrowband noise produces a time envelope that is very irregular compared to that produced by a broadband noise. Thus, both the sinusoidal and narrowband noise yield waveforms which are dissimilar to those produced by a pulsed tonal signal and a continuous broadband noise. With a broadband noise signal and masker, the signal-to-masker phase angle can be controlled, the masker can be continuous and the signal pulsed, and the time-envelope is relatively smooth. Thus, the broadband noise yields a waveform which is similar to that produced by a tonal signal and noise masker. 
The present paper represents a further attempt to clarify the problems raised when the signal-to-masker phase angle is controlled and when the detectability of an interaurally phase-reversed signal is measured. The research consists of two experiments: in the first, both signal and masker are pulsed tones of the same frequency and duration; in the second, the masker is a continuous wideband noise and the signal is a coherent noise of the same bandwidth.

\section{METHOD}

\section{Experiment I}

All data were obtained using a two-alternative temporal forced-choice technique. The trial sequence consisted of a $512-\mathrm{msec}$ waming period, a 512-msec pause, a 128-msec observation interval, a 512 -msec pause, a 128 -msec observation interval, a 1.536-sec response interval, and a $512-\mathrm{msec}$ feedback interval. The warning period was indicated to the listeners by a light. During the feedback interval, one of two lights was iluminated to indicate to the listener which of the two preceding observation intervals contained the signal.

During each of the two observation intervals, a $128-\mathrm{msec}$ $250-\mathrm{Hz} 70-\mathrm{dB}$ SPL tone was gated with a 10 -msec rise and decay time. This tonal masker was presented in-phase at the ears (MO). During one of the two observation intervals, a signal of the same frequency, duration, and rise-decay time was added to the masker at one of three signal-masker phase angles $(\alpha): 0,45$, or 90 deg. The signal was always presented binaurally, but in some conditions it was presented in-phase binaurally (SO) and in others in phase reversal $\left(S_{\Pi}\right)$. The probability of the signal being presented in the first observation interval was equal to that in the second.

For each combination of interaural conditions (MO-SO and MO-S $\Pi$ ) and signal-masker phase angles ( $\alpha=0,45$, and $90 \mathrm{deg}$ ), three signal levels were used. For all conditions other than MO-SO and $\alpha=90 \mathrm{deg}$, the three levels were separated by $2-\mathrm{dB}$ steps. For MO-SO and $\alpha=90 \mathrm{deg}$, the three levels were separated by $1-\mathrm{dB}$ steps.

Four female listeners with clinically normal hearing participated in the experiment. They worked for approximately $1 \mathrm{~h}$ per day for approximately 20 days. Each day consisted of two listening periods separated by a $10 \mathrm{~min}$ rest period. The first session of each day began with a 20-trial practice block during which the signal was elevated by 1 or $2 \mathrm{~dB}$ over the level to be used in the following block. Following the practice block, three experimental sessions were run. After the rest period, the second session of each day consisted of at least two and sometimes three additional experimental blocks. During each session, signal levels were presented in descending order, i.e., highest levels were run first. During any one 80-trial block, the signal level was held constant.

Two of the four listeners were trained initially on MO-SO and the other two on MO-Sח. After training, a minimum of 9 days (18 sessions) were run before the listeners were trained on the remaining conditions. Thus, a minimum of 480 trials wer 2 run for each combination of three levels, three signal-masker angles, and two interaural angles. During training on each intcraural phase condition, the listeners were told to use the feedback lights to help them learn to make their decisions. For the MO-SO condition, they were instructed that their task would be to determine in which of the two intervals the tone sounded "louder." In the MO-S $\Pi$ conditions, they were instructed that their task would be to determine in which of the two intervals the tone sounded "off center." In all of the MO-Sח conditions, the ear that led in phase and/or had the greater amplitude was the left ear.
All phase angles were calibrated according to the electrical inputs to the headphones (Telephonics, TDH-39). Interaural phase relations were measured by observing the Lissajous figure produced on an oscilloscope. Signal-masker phase angles were calibrated by measuring voltage changes at the headphones when the signal was added to the masker.

\section{Experiment II}

A single noise generator provided both the masker and the signal. Signal-masker phase differences were obtained by inserting a Grason-Stadler Model 3520AA phase shifter after the wideband $(100-3,000 \mathrm{~Hz})$ filter. The masker, also wideband filtered, was continuous and had a noise power per unit bandwidth of approximately $50 \mathrm{~dB}$ SPL. The trial sequence was identical to that used in Experiment I except that the two observation intervals were marked for the Ss by indicator lights. Signal-duration and rise-decay time were also the same as in Experiment I. Four different listeners, with clinically normal hearing, were employed. The daily routine for Experiment II Ss was the same as that for the $S s$ in Experiment I. As in Experiment I, $\alpha$ values of 0,45 , and $90 \mathrm{deg}$ were employed when the signal condition was $S O$. When the condition was $S_{\Pi}$, however, $\alpha$ values of $0,45,90$, and $135 \mathrm{deg}$ were employed.

\section{RESULTS}

In both experiments, three-point psychometric functions were obtained for each combination of interaural phase angle and signal-masker phase angle. For each $S$ and each 80-trial block, the quantity $P(C)=$ $1 / 2[P(1 \mid I)+P(2 \mid I I)]$ was computed. In this equation, $P(1 \mid I)$ is the proportion of times the listener correctly detected the presence of the signal in the first observation interval. Similarly, $\mathrm{P}(2$ । II $)$ is the proportion of correct detections for the second observation interval. The average value of $P(C)$ for each condition for each $S$ was plotted against the signal-to-masker ratio, $20 \mathrm{log}$ S/M. A computer was employed to obtain the best fitting values for the transform of the function: $d^{\prime}=$ $\mathrm{m}\left(\mathrm{S}^{2} / \mathrm{M}^{2}\right)^{\mathrm{k}} .1$ The program transformed $\mathrm{d}^{\prime}$ to $\mathrm{P}(\mathrm{C})$ and $\mathrm{S}^{2} / \mathrm{M}^{2}$ to $20 \log \mathrm{S} / \mathrm{M}$ and obtained the values of $\mathrm{m}$ and $\mathrm{k}$ which led to a minimum least squares between $P(C)$ values from the data and those from the equation.

Average psychometric functions for each condition and each experiment are shown in Figs. 1.4.2 These functions are typical in their forms and relative positions of the functions for individual Ss. Of course, some Ss were more sensitive than others, but they did not differ markedly in any other systematic fashion. Figure 1 shows psychometric functions obtained in Experiment I for the three values of $\alpha(0,45$, and $90 \mathrm{deg})$ when the signal condition was $\mathrm{SO}$. These functions indicate that as $\alpha$ is increased from 0 to $90 \mathrm{deg}$, increasing values of $20 \log \mathrm{S} / \mathrm{M}$ are required. Such an increase would be predicted on the basis of the increasing signal amplitude required to maintain a constant power increment. The values of $k$ which best fit the data were 0.5 at $\alpha=0 \mathrm{deg}$, 0.4 at $\alpha=45 \mathrm{deg}$, and 0.9 at $\alpha=90 \mathrm{deg}$. The values of 0.4 and 0.5 are not significantly different from each other, but are different from 0.9. Larger values of $k$ should be obtained as $\alpha$ is increased, since the rate of 
change of incremental power with signal amplitude is greater for larger values of $\alpha$.

Figure 2 shows average psychometric functions obtained with $S_{\Pi}$ signals in Experiment $I$. The $k$ values obtained are: 0.6 at $\alpha=0 \mathrm{deg}, 0.5$ at $\alpha=45 \mathrm{deg}$, and 0.4 at $\alpha=90 \mathrm{deg}$. These differences do not appear to be significant, and individual Ss show no apparent consistency with regard to the relation of $k$ values to $\alpha$. With regard to the horizontal position of the functions, it appears that the $\alpha=0$ deg condition requires a greater signal level than do the $45-$ or $90-\mathrm{deg}$ conditions.

The average psychometric functions for the SO conditions oi Experiment II are shown in Fig. 3. Although they do not indicate differing $\mathrm{k}$ values as did the data of Experiment I, they do show the expected horizontal shift (greater $20 \log \mathrm{S} / \mathrm{M}$ values required for the same level of performance) as $\alpha$ is increased. Thus, the noise-on-noise data, like the tone-on-tone data, agree reasonably well with a power-increment model.

The $S_{\Pi}$ conditions of Experiment II, shown in Fig. 4, indicate no significant change in $\mathrm{k}$ values as a function of $\alpha$. They do show that as $\alpha$ is increased from 0 to $135 \mathrm{deg}$, increasing values of $20 \log \mathrm{S} / \mathrm{M}$ are required.

Figures 5 and 6 summarize the results shown in the psychometric functions by plotting the values of $20 \mathrm{log}$ $\mathrm{S} / \mathrm{M}$ necessary for $\mathrm{P}(\mathrm{C})=0.75$ as a function of $\alpha$. These figures were obtained from the average psychometric functions (Figs. 14) but are not essentially different from comparable functions plotted for individual Ss (i.e., individual Ss show functions of the same general form as is illustrated by the average data). It should be noted that, since the values of $\mathrm{k}$ differ for different values of $\alpha$, the forms of the SO and $S_{\Pi}$ curves of Figs. 5 and 6 may be made to change somewhat by choosing a value of $P(C)$ other than 0.75 . However, such effects appear to be small and, it is felt, will have no effect on the conclusions to be drawn from the data. A second point to be made is that confidence limits on the values of $20 \log$ S/M shown in Figs. 5 and 6 are a function of the values of $k$ which are obtained: low values of $k$ (as in

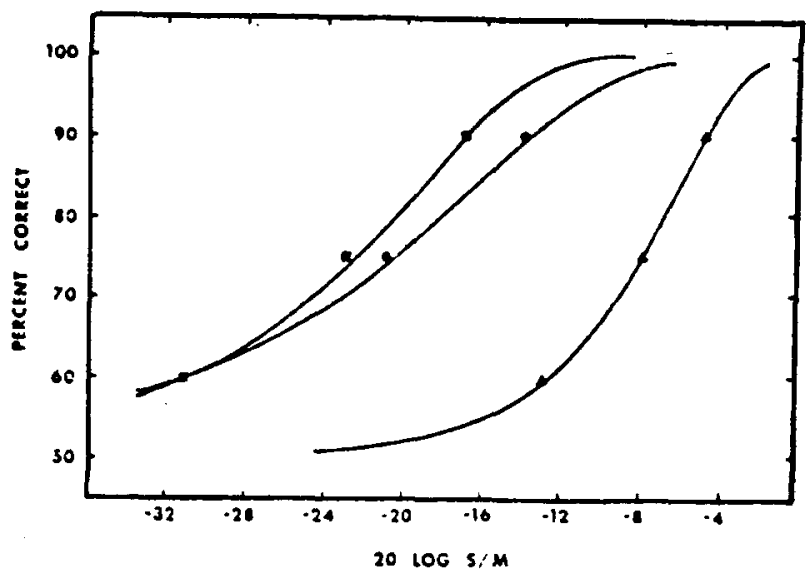

Fig. 1. Average psychometric functions for $S O$ from four $S s$ in Experiment 1. Squares, $\alpha=0$ deg; circles, $\alpha=45$ deg; triangles, $\alpha$ $=90 \mathrm{deg}$.

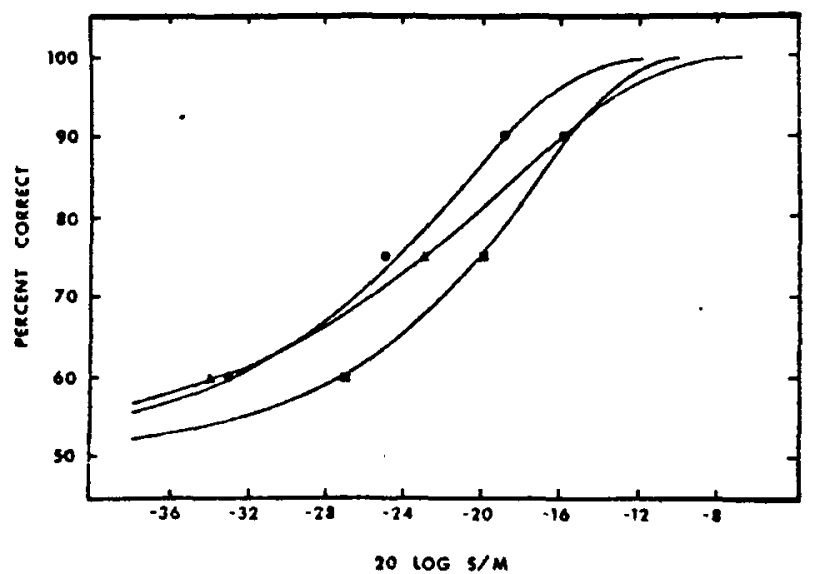

Fig. 2. Average psychometric functions for $S_{\Pi}$ from four $S$ s in Experiment I. Squares, $\alpha=0$ deg; circles, $\alpha=45$ deg; triangles, $\alpha$ $=90 \mathrm{deg}$.

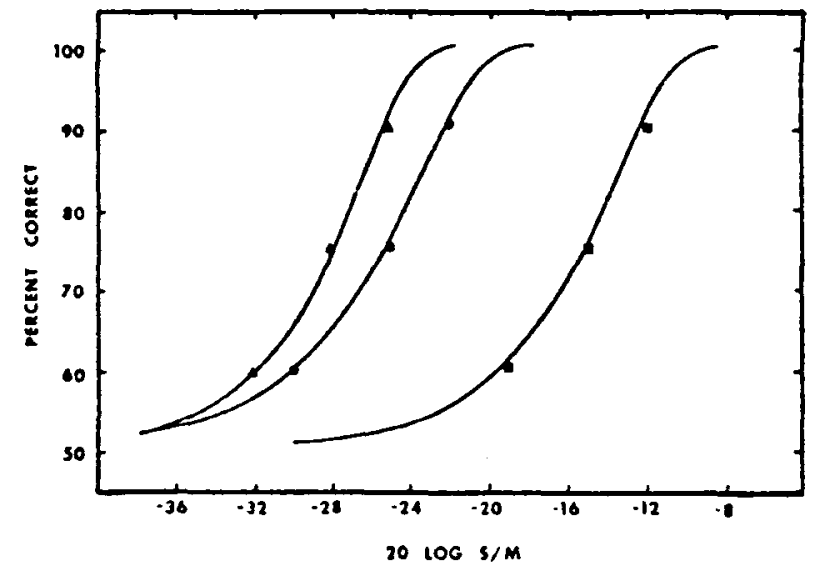

Fig. 3. Average psychometric functions for $S O$ from fcur $S s$ in Experiment II. Triangles, $\alpha=0$ deg; circles, $\alpha=45 \mathrm{deg}$; squares, $\alpha=90 \mathrm{deg}$.

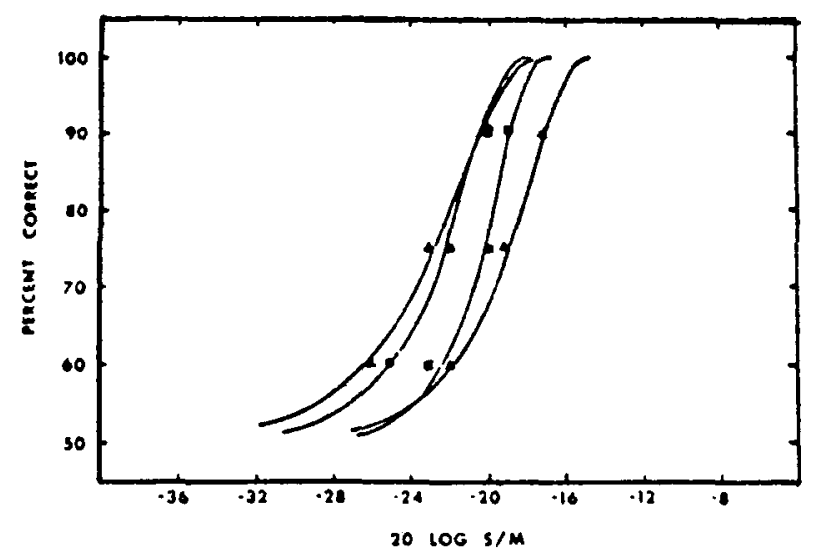

Fig. 4. Average psychometric functions for $S \Pi$ from four $S s$ in Experiment II. Filled triangles, $\alpha=0$ deg; circles, $\alpha=45 \mathrm{deg}$; squares, $\alpha=90$ deg; triangles, $\alpha=135 \mathrm{deg}$.

Experiment I) will lead to more variability of $20 \log$ S/M than will high values.

The smooth curves drawn through the SO data in Figs. 5 and 6 are based upon a constant power-increment 


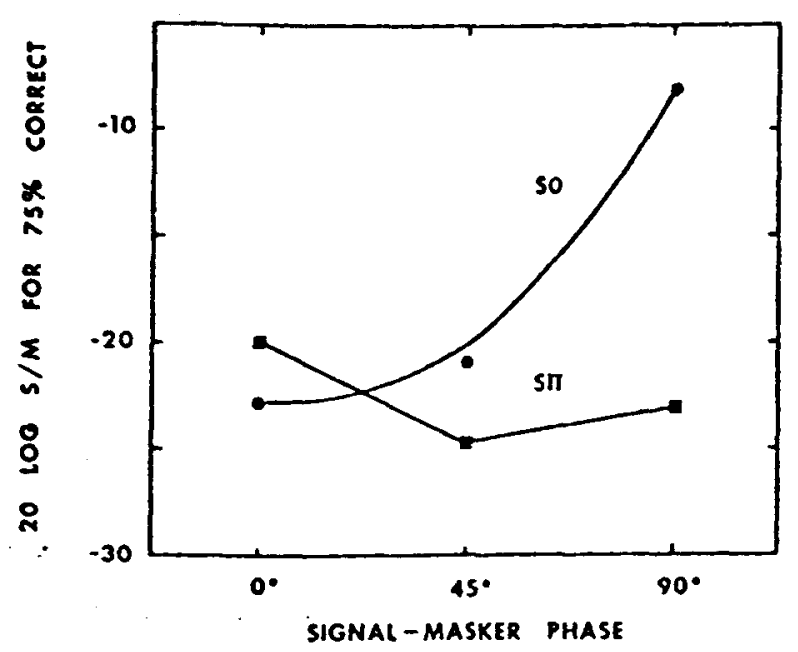

Fig 5. Average data from four Ss in Experiment l. Squares, Srl: circles, SO. The smooth curve drawn through the SO data is based on the constant power-increment hypothesis.

hypothesis. The fit to the data is excellent. The average power increment in Experiment $\mathrm{I}$ is $0.6 \mathrm{~dB}$. For Experiment II, the cerresponding value is $0.5 \mathrm{~dB}$. The dashed line in Fig. 6 is an extrapolation of the power-increment hypothesis to $135 \mathrm{deg}$ (a value not experimentally determined). The upper triangle denotes the value of $20 \log \mathrm{S} / \mathrm{M}$ necessary to obtain a power increment of $0.5 \mathrm{~dB}$; the lower one denotes that necessary to obtain a $0.5-\mathrm{dB}$ power decrement.

The $S_{\Pi}$ data of Experiment I show a somewhat U-shaped function with the largest value of $20 \log \mathrm{S} / \mathrm{M}$ occurring at $\alpha=0 \mathrm{deg}$ and lower values at $\alpha=45 \mathrm{deg}$ and at $\alpha=90 \mathrm{deg}$. Each of the functions for individual Ss were of the same form.

The Experiment II $S_{\Pi}$ conditions show a slight increase in $20 \log \mathrm{S} / \mathrm{M}$ as $\alpha$ is increased from 0 to $135 \mathrm{deg}$. The increase is approximately $1 \mathrm{~dB}$ per $45 \mathrm{deg}$. This trend was also reflected in the data for individual Ss.

Both Experiment $I$ and Experiment II show $S_{\Pi}$ detectability to be better (smaller values of $20 \log S / M$ ) than SO except at $\alpha=0 \mathrm{deg}$, where Experiment I shows a decrement of about $3 \mathrm{~dB}$ and Experiment II, a decrement of about $1 \mathrm{~dB}$.

\section{DISCUSSION}

\section{In-Phase Signals}

The results of both experiments for the conditions in which there were no interaural discrepancies (the SO conditions) are fit well by a constant power- or a constant energy-increment model and are, therefore, consistent with most theories of signal detectability. For Experiment I, the average power increment required for a $P(C)$ of 0.75 was approximately $0.6 \mathrm{~dB}$. The corresponding figure for Experiment II was approximately $0.5 \mathrm{~dB}$. These values agree well with classical data (see Riesz, 1928; Green, 1960). In the present experiments, the SO data represent control conditions and are used only to compare antiphasic with homophasic detection. In light of observations made by Wightman (1971), it should be pointed out that the good fit of the SO data in Experiment I to a constant energy-increment function may be taken as evidence that there was no contamination by energy spread to frequencies other than those of the signal and masker. This problem was avoided by gating both signal and masker simultaneously. For Experiment II, where the masker was continuous, the problem of energy spread would seem to be negligible, since both signal and maskers were wideband noises. This is borne out by the fact that the SO data of Experiment II are also fit well by the constant energy-increment hypothesis.

\section{Phase-Reversed Signals}

\section{Lateralization}

It is important to remember that the task of the Ss was to detect the presence or absence of the signal, not to lateralize the image of the masker or signal plus masker. The task of lateralization under conditions such as those described here has been extensively investigated by Jeffress and McFadden and by McFadden, Jeffress, and Ermey. However, the cues usually associated with the task of lateralization (interaural temporal and interaural intensive differences) are present in the $S_{\Pi}$ conditions, and the data may be used to estimate "thresholds" for the use of these cues in the detection task. The interaural temporal difference, $\Delta t$, may be estimated from the data obtained for $\alpha=90 \mathrm{deg}$, since at this value the interaural intensive difference is zero and

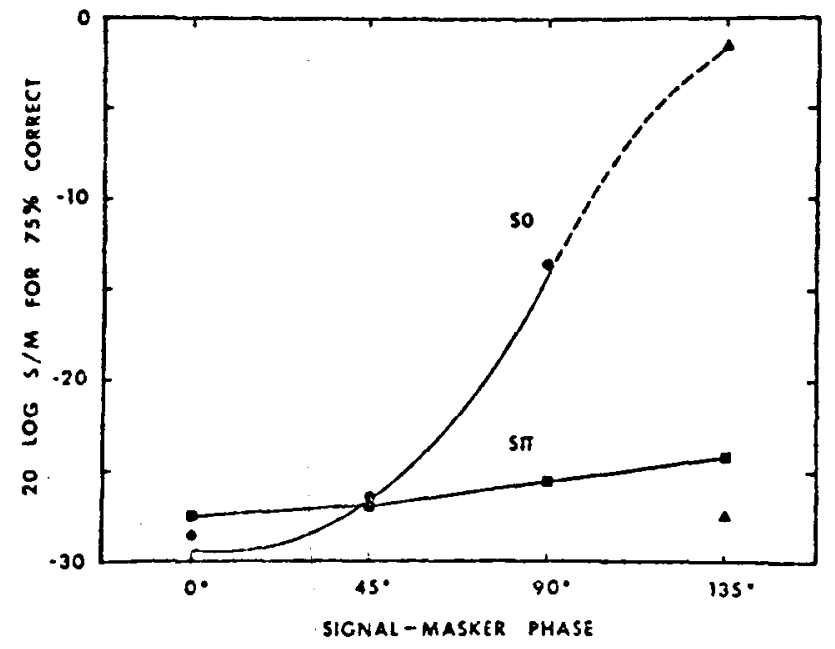

Fig. 6. Average data from four $S s$ in Experiment II. Squares, $\mathrm{S}_{\Pi}$; circles, SO; triangles, predicted values for $\mathrm{SO}$ at $\alpha=135 \mathrm{deg}$. The smooth curve drawn through the So data is based on the constant power-increment hypothesis. The dashed portion of the curve is an extrapolation for the constant power-increment hypothesis. 
the monaural power increment is negligible. For Experiment $\mathrm{I}$, the average $\Delta^{\mathrm{t}}$ was approximately 90 microsec for the $250-\mathrm{Hz} 128-\mathrm{msec}$ tone. This value is in the range of estimates of the interaural temporal-difference threshold reported by other researchers. For example, Hafter and Carrier (1970) reported 200 microsec for an untrained $S$ and 36 microsec and 112 microsec for highly trained Ss. Yost $(1972 \mathrm{a}, \mathrm{b})$ reported $60-70 \mathrm{mic}$ rosec for $500 \mathrm{~Hz}$ and 128 microsec for $250 \mathrm{~Hz}$. Garner and Wertheimer (1951) obtained a value of 170 microsec for $200 \mathrm{~Hz}$ at $90 \mathrm{~dB}$ SPL and 350 microsec at $50 \mathrm{~dB}$. Other investigators (Klumpp \& Eady, 1956; Zwislocki \& Feldman, 1956; Hershkowitz \& Durlach, 1969) have found smaller values. At $65 \mathrm{~dB}$ SPL, Klumpp and Eady reported 27 microsec for $250 \mathrm{~Hz}$. Zwislocki and Feldman report approximately 30 microsec for a range of SLs from $10 \mathrm{~dB}$ to $90 \mathrm{~dB}$ and a frequency of $250 \mathrm{~Hz}$, while Hershkowitz and Durlach report 11.7 microsec at $500 \mathrm{~Hz}$. Since Experiment II involved phase shifting a wideband noise, interaural temporal differences cannot be estimated.

When $\alpha$ is $0 \mathrm{deg}$ there is an interaural intensive difference, $\Delta \mathrm{I}$. For Experiment $I$, the average value of $\Delta^{I}$ was $1.8 \mathrm{~dB}$, a value which is considerably greater than the $0.8 \mathrm{~dB}$ reportid by Mills (1960) for a $250-\mathrm{Hz}$ tone. Wightman (1971) reports a $\Delta^{I}$ of $1.3 \mathrm{~dB}$ at $262 \mathrm{~Hz}$, Hafter and Carrier show an average $\Delta \mathrm{I}$ of about $0.9 \mathrm{~dB}$, and Yost $(1972 \mathrm{a}, \mathrm{b})$ reports an average of $1.0 \mathrm{~dB}$. The interaural $\Delta I$ estimated from the data of Experiment II is about $1.29 \mathrm{~dB}$. For a narrowband signal and noise centered at $500 \mathrm{~Hz}$, Jeffress and McFadden report an interaural $\Delta^{\mathrm{I}}$ of $1.0 \mathrm{~dB}$. McFadden, Jeffress, and Ermey report a $\Delta \mathrm{I}$ of $1.42 \mathrm{~dB}$ for a narrowband noise centered at $250 \mathrm{~Hz}$. Thus, the interaural intensive differences obtained in both Experiments I and II are in reasonably good agreement with other relevant data.

\section{Detectability and $\alpha$}

The data of Experiment I, showing best detectability at a signal-to-masker phase angle of $45 \mathrm{deg}$, preclude both the Webster-Jeffress model and the E-C model. These data are similar to the $262-\mathrm{Hz}$ data reported by Wightman (1971) and by Yost (1972b) and to the data for some of the Ss in the Hafter and Carrier experiments at 500 and $1,000 \mathrm{~Hz}$, but not at $250 \mathrm{~Hz}$. Although McFadden, Jeffress, and Ermey do not report means across Ss, we have taken the liberty of averaging their data for the $250-\mathrm{Hz}$ center frequency noise condition. These average data compare favorably in the form of the $S_{\Pi}$ function with the data from Experiment $I$. The data of Experiment II, which show that, for noise, the effect of signal-to-masker phase angle is to decrease detectability with increasing values of $\alpha$, allow us to reject the Webster-Jeffress hypothesis, but leave some doubt about the E-C model. The departure from a horizontal line is slight. These data are similar in form to average data based on the individual data reported by Jeffress and McFadden (1971), who employed a narrowband noise centered at $500 \mathrm{~Hz}$.

It is important to note that in both Experiments I and II, antiphasic detection at $\alpha=0 \mathrm{deg}$ is worse than homophasic detection at the same angle. In only two of the seven experiments we have described is this the case: Wightman (1969) and Jeffress, Blodgett, Sandel, and Wood. In the study by Yost (1972b), he reported that at $\alpha=0 \mathrm{deg}$ antiphasic and homophasic detection were about equal. However, Yost reported that detection in the SM condition at $\alpha=0$ deg was, on the average, $5 \mathrm{~dB}$ worse than that in the SO or $\mathrm{S}_{\Pi}$ conditions. Yost (1972b) also showed that the interaural intensive difference required at $\alpha=0$ deg in both the $S_{\Pi}$ and $S M$ conditions was about $1.0 \mathrm{~dB}$. In our case, the average decrement in performance for $S_{\Pi}$ at $\alpha=0$ deg was about $3 \mathrm{~dB}$ for Experiment I and about $1 \mathrm{~dB}$ for Experiment II. This decrement in performance for $S_{\Pi}$ relative to SO may be interpreted in two ways: our Ss were relatively insensitive to interaural intensive differences (the cue available at $\alpha=0 \mathrm{deg}$ for $\mathrm{S}_{\Pi}$ ) or they were quite sensitive to the power increment (the cue available at $\alpha=0 \mathrm{deg}$ for SO). A comparison of our data with those presented in the several relevant papers suggests that a combination of these two factors will explain the decrement. In Experiment I, our Ss were extremely sensitive to the power increment, requiring only about $0.6 \mathrm{~dB}$ for SO at $\alpha=0 \mathrm{deg}$. [Hafter and Carrier report values of $0.95,0.75$, and $0.90 \mathrm{~dB}$ for three Ss at $250 \mathrm{~Hz}$; Wightman (1971) shows data which indicate a required power increment of about $0.75 \mathrm{~dB}$; Yost (1972a) reports power increments of approximately $0.7 \mathrm{~dB}$ over the $12-\mathrm{dB}$ intensity range which he investigated.] For data from experiments with noise as a masker and as a signal, the discrepancy is even greater. The Ss in Experiment II required an increment of only $0.5 \mathrm{~dB}$, while Jeffress and McFadden report power increments ranging from 1.73 to $2.00 \mathrm{~dB}$ and McFadden et al report values ranging from 2.92 to $3.86 \mathrm{~dB}$. Thus, in both Experiments I and II, our Ss seem extremely sensitive to the power increment available with SO at $\alpha=0 \mathrm{deg}$. The situation for the $S_{\Pi}$ signals at $\alpha=0 \mathrm{deg}$ is quite different, at least in Experiment I. The $\Delta \mathrm{I}$ of $1.8 \mathrm{~dB}$ required by our $\mathrm{Ss}$ indicates that they are relatively less sensitive to interaural intensive differences than $S s$ in other experiments. Hafter and Carrier report data indicating an average interaural intensive difference of about $0.6 \mathrm{~dB}$ at $250 \mathrm{~Hz}$, Wightman (1971) shows data indicating about $1.3 \mathrm{~dB}$, and Yost $(1972 \mathrm{a}, \mathrm{b})$ reported $1.0 \mathrm{~dB}$ at 250 , 500 , and $3,000 \mathrm{~Hz}$ and for a $12-\mathrm{dB}$ range in tonal masker intensity. With respect to the noise data, our Ss, requiring an average interaural difference of $1.29 \mathrm{~dB}$, fall near the range ( 0.8 to $1.13 \mathrm{~dB}$ ) reported by Jeffress and McFadden (1971) and within that $(1.07$ to $1.80 \mathrm{~dB})$ reported by McFadden et al (1971).

The role of sensitivity (and/or detectability) to 
homophasic conditions in determining the relative advantage of antiphasic to homophasic detection has been discussed by others. As Jeffress, Blodgett, Sandel, and Wood pointed out, "... any condition which requires a large signal for monaural detection has the potentiality for a substantial MLD under antiphasic conditions." Apparently, for tone-on-tone and wideband noise-in-noise conditions, the sensitivity of the "monaural" system to a power increment is so great that the binaural system has little room to show an improvement. For narrowband noise, however, Ss are relatively insensitive to the power increment (perhaps due to the relatively large fluctuations in the envelope of the narrowband noise), and the binaural system has room to show an improvement. The fact that the binaural system is not adversely affected by the narrowband noise is shown by the good agreement of the interaural intensive differences obtained in Experiment II with those obtained by Jeffress and McFadden and by McFadden et al.

The data of Experiment I are extremely well fit by the "lateralization" model of Hafter and Carrier. The average error in predicting the value of $20 \log \mathrm{S} / \mathrm{M}$ for $\alpha$ $=45 \mathrm{deg}$ (the one point to be predicted) is about $0.75 \mathrm{~dB}$. Since a wideband noise was used in Experiment II, interaural temporal differences cannot be computed, and thus the "lateralization" model cannot be applied.

Although Ss within each of the two experiments showed essentially the same functions relating signal level to $\alpha$ (for $0 \leqslant \alpha \leqslant 90 \mathrm{deg}$ ), a comparison of the two experiments showed quite different functions. Experiment I shows best performance at $\alpha=45 \mathrm{deg}$, while Experiment II showed best performance at $\alpha=$ 0 deg. Functions similar to each of these appear in other papers. An evaluation of the "lateralization" model proposed by Hafter and Carrier indicates that the model is capable of predicting functions over the range $0 \leqslant \alpha \leqslant 90$ deg which are monotonically increasing with increasing values of $\alpha$, are monotonically decreasing, or are U-shaped. Which function is obtained will be determined by the relative sensitivity of the $S$ to interaural temporal difference and to interaural intensive difference. As was pointed out previously, the "lateralization" model provides a poor fit to the data for $90<\alpha<180 \mathrm{deg}$. What we are forced to conclude, then, is that the "lateralization" model provides a better fit to the data than does either the Webster-Jeffress model or the E-C model. However, the "lateralization" model does not appear to be a complete description of what occurs in detection tasks involving coherent signals and maskers.

\section{REFERENCES}

Durlach, N. I. Binaural signal detection: Equalization and cancellation theory. In J. V. Tobias (Ed.), Foundations of modern auditory theory. Vol. II. New York: Academic Press, 1972. Pp. 371-462.

Garner, W. R., \& Wertheimer, M. Some effects of interaural phase difference on the perception of pure tones. Journal of the Acoustical Society of America, 1951, 23, 664-667.

Green, D. M. Auditory detection of a noise signal. Journal of the Acoustical Society of America, 1960, 32, 121-131.

Green, D. M., \& Henning, G. B. Audition. Annual Review of Psychology, 1969, 20, 105-128.

Hafter, E. R. Quantitative evaluation of a lateralization model of masking-level differences. Journal of the Acoustical Society of America, 1971, 50, 1116-1122.

Hafter, E. R., \& Carrier, S. C. Masking-level differences obtained with a pulsed tonal masker. Journal of the Acoustical Society of America, 1970, 47, 1041-1047.

Hershkowitz, R. M., \& Durlach, N. I. Interaural time and amplitude jnds for a $500-\mathrm{Hz}$ tone. Journal of the Acoustical Society of America, 1969, 46, 1464-1467.

Hirsh, I. J., \& Webster, F. A. Some determinants of interaural phase effects. Journal of the Acoustical Society of America, $1949,21,495-501$.

Jeffress, L. A. A place theory of sound localization. Journal of Comparative \& Physiological Psychology, 1948, 41, 35-39.

Jeffress, L. A., Blodgett, H. C., Sandel, T. T., \& Wood, C. L., III. Masking of tonal signals. Journal of the Acoustical Society of America, 1956, 28, 416-426.

Jeffress, L. A., \& McFadden, D. Differences of interaural phase and level in detection and lateralization. Journal of the Acoustical Society of America, 1971, 49, 1169-1179.

Klumpp, R. G., \& Eady, H. R. Some measurements of interaural time difference thresholds. Journal of the Acoustical Society of America, 1956, 28, 859-860.

Levitt, H., \& Lundry, E. A. Some properties of the vector model for binaural hearing. Journal of the Acoustical Society of America, 1966, 39, 1232(A).

Licklider, J. C. R. Influence of interaural phase relations upon the masking of speech by white noise. Journal of the Acoustical Society of America, 1948, 20, 150-159.

McFadden, D., Jeffress, L. A., \& Ermey, H. L. Differences of interaural phase and level in detection and lateralization: $250 \mathrm{~Hz}$. Journal of the Acoustical Society of America, 1971, 50, 1484-1493.

Mills, A. W. Lateralization of high frequency tones. Journal of the Acoustical Society of America, 1960, 32, 132-137.

Riesz, R. R. Differential intensity sensitivity of the ear for pure tones. Physics Review, 1928, 31, 867-875.

Rilling, M. E., \& Jeffress, L. A. Narrow-band noise and tones as signals in binaural detection. Journal of the Acoustical Society of America, 1965, 38, 202-206.

Robinson, D. E. Some computations relevant to the Webster-Jeffress model for binaural signal detection. Journal of the Acoustical Society of America, 1966, 39, 1232(A).

Webster, F. A. Influence of interaural phase on masked thresholds. I. The role of interaural time-deviation. Journal of the Acoustical Society of America, 1957, 23, 452-462.

Wightman, F. L. Binaural masking with sine-wave maskers. Journal of the Acoustical Society of America, 1969, 45, 72.78 .

Wightman, F. L. Detection of binaural tones as a function of masker bandwidth. Journal of the Acoustical Society of America, 1971, 50, 623-636.

Yost, W. A. Tone-on-tone binaural masking. Unpublished $\mathrm{PhD}$ thesis, Indiana University, 1970.

Yost, W. A. Weber's fraction for the intensity of pure tones presented binaurally. Perception \& Psychophysics, 1972a, 11, $61-64$.

Yost, W. A. Tone-on-tone masking for three binaural listening conditions. Journal of the Acoustical Society of America, $1972 \mathrm{~b}, 52,1234-1237$.

Zwislocki, J., \& Feldman, R. S. Just noticeable difference in 
dichotic phase. Journal of the Acoustical Society of America, $1956,28,860-864$.

\section{NOTES}

1. The choice of this equation is made on purely empirical grounds. It is not our intent to suggest that the equation represents the proper rational function to describe data of the sort reported here. Rather, this equation allows us to conveniently manipulate the data in order to obtain transforms such as those shown in Figs. 5 and 6 . Further, it is felt that the fits obtained with this equation are excellent and that any error introduced because this is the "wrong" function will be small.

2. Averaging was done by fitting the data for each listener using the technique described previously; obtaining, from the best-fitting equation for each $S$, the $20 \log S / M$ values necessary for $0.60,0.75$, and 0.90 percent correct; averaging corresponding $20 \log \mathrm{S} / \mathrm{M}$ values over Ss; and fitting the average values using the same curve-fitting technique as was used for the individual Ss.

(Received for publication June 28, 1973; accepted September 21, 1973.) 